\title{
EFFECT OF INLET PROBE SUPPORT ON UNSTEADY AERODYNAMIC PERFORMANCE OF SINGLE-STAGE TRANSONIC COMPRESSOR
}

\author{
Rongfei Yang \\ Nanjing University of Aeronautics and \\ Astronautics \\ yrf@nuaa.edu.cn \\ Nanjing, Jiangsu, China \\ Haixu Liu \\ Nanjing University of Aeronautics and \\ Astronautics \\ 774110548 @qq.com \\ Nanjing, Jiangsu, China
}

\author{
Yan Xue \\ Nanjing University of Aeronautics and \\ Astronautics \\ 1125560713@qq.com \\ Nanjing, Jiangsu, China
}

\begin{abstract}
Involved in compressor performance testing, an inlet probe support causes flow blockage and produces shedding vortex, which affects aerodynamic performance of the compressor. In this paper a single-stage transonic compressor NASA Stage 35 is taken as an example, and a cylindrical probe support with $10 \mathrm{~mm}$ diameter is located one chord away from the rotor leading edge. Steady and unsteady flow simulation for the compressor stage with and without the support is carried out by commercial CFX software tool. Results show that the support brings about substantial performance degradation of compressor. Meanwhile, the characteristics of the vortex shedding from the support and the flow field structure of compressor change by the interaction of the support and the compressor. The shedding vortex frequency of probe support is affected by the working condition of the compressor. Under the strong interaction of probe support and transonic rotor leading edge shock, the shedding vortex frequency of probe support is locked by the rotor blade passing. Wider distance between the support and leading edge of the rotor gives rise to homologous change of eddy phase in the rotor.
\end{abstract}

\section{INTRODUCTION}

In the aerodynamic performance test for compressors, the inlet total pressure probes are usually circumferentially arranged evenly and are located $0.3-1.0$ times the axial chord length upstream of the first blade row (Zhang Baocheng, 2005). With the increase in number and diameter of probe supports, the unsteady wakes and blockage effect of inlet probe supports inevitably affect the flow field into the compressor. Thus, research on this topic is helpful to reasonably evaluate and revise the influence of the probe support on the aerodynamic performance of the compressor.

Many scholars have investigated the effects of inlet probe support on aerodynamic performance of compressors in the past few decades. Wyler (1975) studied the blockage effect of cylindrical probe supports in the channel. His results show that the blockage effect is the most serious in Mach number range from 0.8 to 1.2. Lepicovsky's (2008) experimental studies showed that the rotational probes mounted on the hub made the flow field at the rotor exit of a low-speed axial compressor even worsen .Ma H et.al(2014) experimentally investigated the influences of the stationary probes installed on the casing at the leading edge of rotor on the rotor's exit flow field of a low-speed axial compressor. It was found that the mass flow in the region where the probes have a large influence on is up to $10 \%$ less than that without probes. Meanwhile, parameters such as total pressure rise coefficient and radial velocity coefficient in the measuring planes obviously changed in radial direction. Louis (1976) experimentally observed (using LDV, Laser Doppler Velocimetry) that blockage effect of inlet probes made the velocity downstream of annular turbine stator cascade decrease. Coldrick (2002) numerically studied the effect of probe supports on a single-stage low speed axial compressor. The steady calculations reveal that the mass flow of the stator passages with the blockage effect of probes reduces by about $20 \%$ compared to other adjacent stator passages. 
The probes close to the stators lead to a decrease in the velocity and an increase in the pressure of airflow. Furthermore, the unsteady solutions show that the total pressure near the probes decreases by $150 \mathrm{~Pa}$ and the flow angle increases by 3.5 degrees at the peak point.

Based on studies above, the probes have a significant effect on the internal flow field of the compressor, and their influence is directly related to the factors such as Mach number, blockage ratio and so on. Yang et.al (2017) studied the effects of shapes, axial positions and blockage effect of probe supports on the aerodynamic performance of 1.5-stage compressor with inlet guide vanes. Their findings indicate that the Inlet Guide Vanes (IGVs) can greatly weaken the influence of upstream wakes of the probe supports on the flow field of the downstream compressor. However, the transonic compressors without IGVs frequently come up in the experimental tests. Comparing with compressors with IGVs, the axial distance between the probe support and the rotor leading edge is smaller, which contributes to stronger unsteady interaction between the support wake and the transonic compressor. Therefore, unsteady simulation on a single-stage transonic compressor (NASA Stage35) is conducted in this paper to investigate the interaction of probe support and compressor.

\section{RESEARCH OBJECTS AND CALCULATION APPROACHES}

\section{Research Objects}

To investigate the effects of probe supports on a typical transonic compressor without IGVs, a NASA Stage 35 is adopted in this paper. The geometry and performance data are gathered from Ref. (Reid L. et.al, 1978). The NASA Stage 35 is composed of a 36-blade rotor and a 46-blade stator. The overall NASA Stage 35 performance at design point (corrected mass flow of $20.19 \mathrm{~kg} / \mathrm{s}$ and rotational speed of $17188 \mathrm{RPM}$ ) is a total pressure ratio equal to 1.82 and a stage adiabatic efficiency equal to 0.828 . The tip clearance at design speed is $1 \%$ of the blade height.

In experiments for the compressor, the total pressure/temperature measurement probe mainly consists of probe heads and support. Figure 1 depicts a comb-type probe used in total pressure testing. It can be seen that the more heads the probe has, the larger cross-sectional area of the probe support is. Since the small probe heads are located at upstream of the probe support, the width and intensity of the probe wake are primarily determined by the probe support. Therefore, more attention should be paid on the support rather than heads of the probe and its interaction with the compressor downstream. A $10 \mathrm{~mm}$ diameter probe support is sometimes used in compressor experiments, according to the staffs in compressor laboratory. Xiang et.al (2017) studied the effect of wakes produced by $10 \mathrm{~mm}$ diameter probe support on the performance of compressor cascades. Thus, a cylindrical support with a diameter of $10 \mathrm{~mm}$ is designed in this paper. The support is located one chord away from the rotor leading edge.

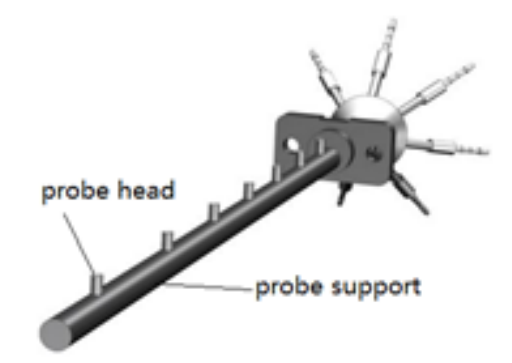

Figure 1 Sketch of a comb-type probe

To reduce unsteady computational efforts, only one-twelfth section of the full geometry is modelled. The computational domain of the compressor without probe supports consists of three rotor blades and four stator blades by increasing the number of stator blades up to 48 and remaining rotor blades unchanged. Meanwhile, 12 inlet probe supports with a diameter of $10 \mathrm{~mm}$ are designed. Therefore, only one probe support is involved in the computational domain of compressor with probe supports. Ratio of blockage area of the supports to the compressor inlet area is 0.08163 . In Fig. 2 a sketch of the computational domain with and without the probe support is reported respectively.

\section{Numerical Method}

NUMECA AutoGrid5, a commercial software tool, is used to generate the grids for the calculation, involving an $\mathrm{O} 4 \mathrm{H}-$ type structured grid and O-grid near the blade wall. The meshing density near the wall is refined to ensure that the y plus of the first mesh normal to the wall is about 2. The grid number of single passage rotor is 850,000 , and that of stator is 690,000 . The total number of grid elements is approximately 4,830,000 and 6,980,000 for the compressor with and without the support respectively. The grid elements in circumferential direction are evenly distributed with minor difference in size at both side of the interface of rotational and stationary computational domains so as to reduce the numerical error there. The numerical grids of global view and partial magnification at interface of upstream and downstream calculation domain are shown in Fig.2. 
Numerical simulations were carried out using the commercial CFD code ANSYS CFX 13.0. The code solves the 3D Reynolds-averaged form of Navier-Stokes equations by using a finite-element based finite-volume method. A second-order high-resolution advection scheme is adopted. The turbulence models used in the calculations is the SST model without considering transition flow. The total pressure, total temperature and flow angle are imposed at the inflow boundary. The inlet total pressure and total temperature are given at 101,325 Pa and $288.15 \mathrm{~K}$ respectively. The inlet turbulence intensity remains at $5 \%$. An average relative static pressure is set at the outflow boundary. On the solid wall boundary, the no-slip and adiabatic conditions are used. Rotational periodic boundary conditions are applied to the lateral surfaces of the flow domain. A mixing plane approach imposed at the rotor-stator interface in steady calculations, whereas a sliding interface technique was applied in unsteady calculations.

A second-order backward Euler transient scheme is used for the time accurate solutions. The steady results were used to provide an initial solution. The physical time step is obtained by Eq. (1) and the maximum inner iteration steps is set as 10. The choice of time step means that the unsteady calculation used 40 time steps per rotor blade passing, 30 time steps per stator blade passing and 120 time steps per probe support passing.

$$
\Delta t=60 \mathrm{~s} /(17188 \mathrm{rpm} \times 120 \times 1 \times 3 \times 4) \approx 2.42 \mu \mathrm{s}
$$

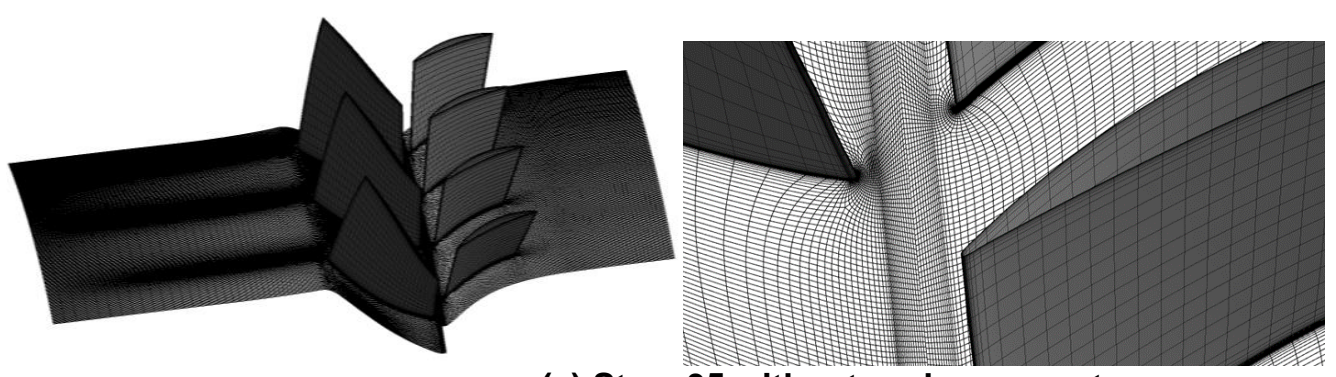

(a) Stage35 without probe support

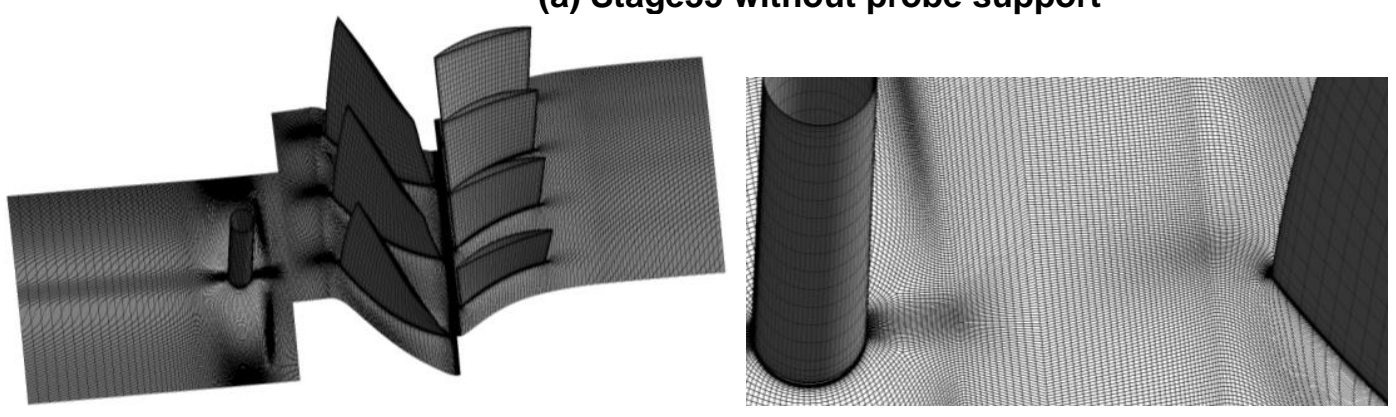

(b) Stage 35 with probe supports

Figure 2 Grids for Stage35 (left: global view, right: partial magnification)

\section{Validity of Numerical Method}

In order to verify the effectiveness of the numerical model, the steady simulation is performed using single passage model for the number of stator unchanged and reduced. Different points on the compressor performance map were obtained by means of a series of outlet static pressure values until the near-stall region reached. The calculated and experimental performance maps at $100 \%$ design speed are presented in Fig.3.

It can be seen that the simulation result of compressor with stator unchanged reasonably agree well with the experimental data near the design point. Errors between the calculation and the measurement are within $3 \%$ for pressure ratio and $1 \%$ for adiabatic efficiency over the whole mass flow range except at the choke point (the error of the efficiency reach the maximum value $2 \%$ ). According to the error analysis data by Morini, M. et.al (2010), the measurement error band of total pressure ratio, total temperature ratio, and efficiency was fixed at $\pm 3 \%$. Thus, the numerical model referenced above can be considered reliable.

When the number of stator blades increased from 46 to 48 , the compressor efficiency and total pressure ratio decrease $2 \%$ and 0.05 respectively at peak efficiency point. In spite of the deviation, the unsteady flow mechanism of the interaction between probe support and compressors does not change because the number of stator blades increase less than $5 \%$. Therefore, a compressor with reduced blade number can be used for unsteady study in this paper. 

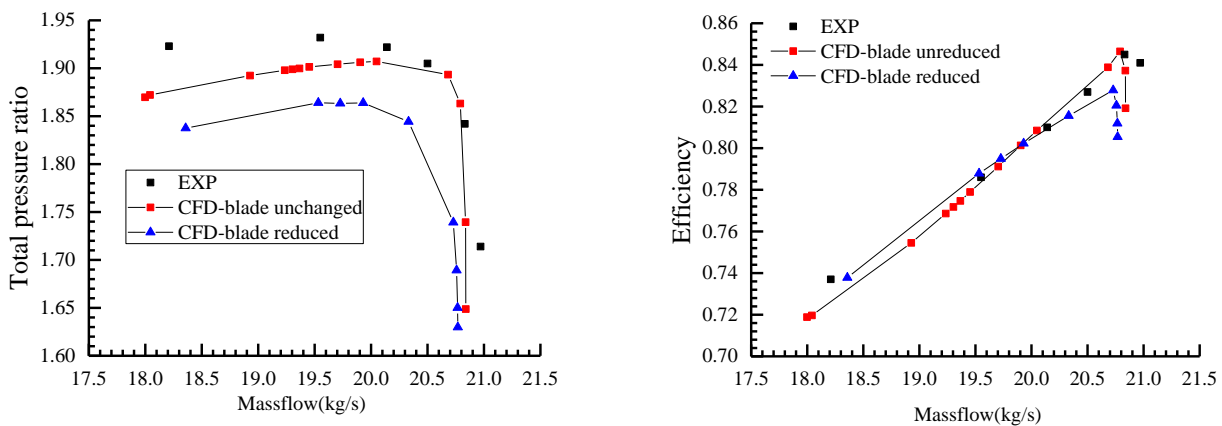

Figure 3 Performance map at $100 \%$ designed speed for Stage 35 without probe

\section{RESULTS AND DISCUSSIONS}

\section{Overall Performance}

The overall performance of the compressor is calculated from inlet plane and outlet plane of computation domain. The steady and unsteady time-averaged performance of the compressor with supports is shown in Fig.4. The steady result of compressor without supports is also added in Fig.4. It can be seen that the steady performance curve of the compressor with supports shifts to the down left compared to that of compressor without supports. The increased blockage due to supports is the primary factor in deteriorating compressor performance. In addition, the steady and unsteady performance of the compressor with probe is very close, which proves the feasibility of the numerical simulation method mentioned above. The total pressure ratio and efficiency of the unsteady solutions are slightly lower than that of steady solutions, especially near the stall point. This trend is consistent with that described by Van Zante et.al (2008).
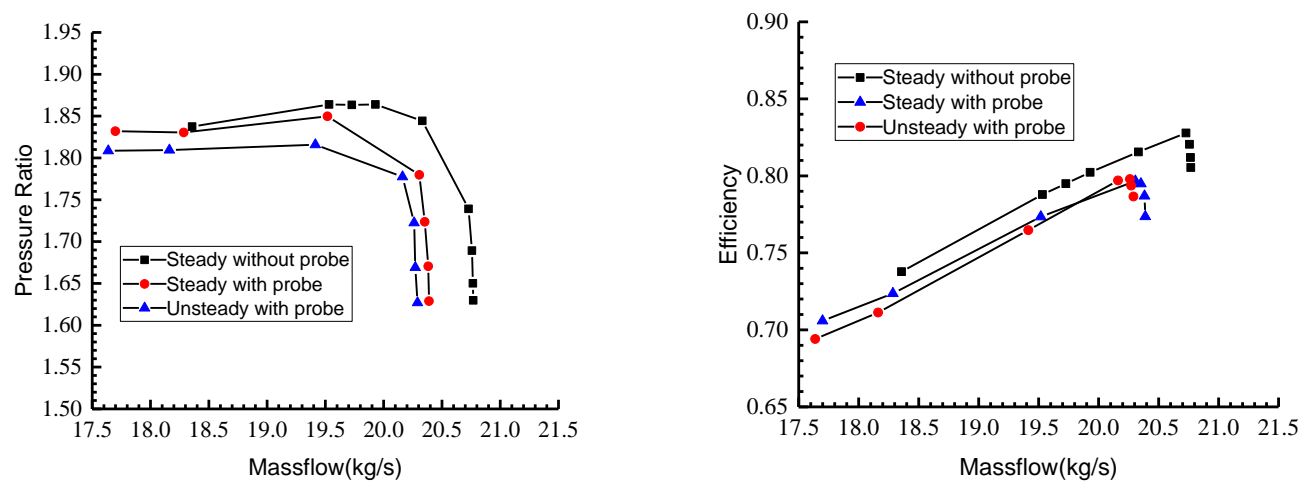

Figure 4 Performance map for compressor with probe supports

\section{Shedding Vortex Frequency of Probe Supports}

A static pressure monitoring point is set on the midspan surface and located 0.5 times the rotor blade chord length downstream of the probe support. The shedding vortex frequency of probe support is obtained from the spectrum analysis of static pressure, as shown in Fig.5. Symbols A1-A7 respectively correspond to the calculating point of compressor working from choke to stall in Fig.4. In addition, the frequencies of $4000 \mathrm{~Hz}, 8100 \mathrm{~Hz}$ and $10321 \mathrm{~Hz}$ are marked with purple line, blue line and red line respectively in Fig.5. The $10321 \mathrm{~Hz}$ corresponds to the rotor blade passing frequency. The $4000 \mathrm{~Hz}$ is close to the probe support shedding vortex frequency calculated by assuming $\mathrm{St} \approx 0.2$, and the $8100 \mathrm{~Hz}$ is nearly two times of probe support shedding vortex frequency. As shown in Fig.5, with the decreasing mass flow of compressor throttled from Point A1 to point A7, the shedding vortex frequency of probe support decrease and the rotor passing frequency remain unchanged. Meanwhile, the main frequency in Fig.5 varies with the working state of the compressor. At the near-stall points, from point A5 to point A7, the main frequency is the rotor blade passing frequency, which is also the shedding vortex frequency of probe support. It is consistent with the results found by Nolan et.al (2011). He believed that the shedding vortex frequency of upstream stator is affected by transonic rotor and locked to rotor blade passing frequency when the gap between stator and rotor is small. As can be seen from Fig.6, strong interaction occurs between the probe support and rotor under the impact of the detachment of the leading-edge shock at Point A5 and Point A7. As a comparison, the intensity of the shock wave at the rotor leading edge is weaker at the choke point A1. In this case, the probe support wake vortex shedding is less affected by the compressor and the shedding frequency is nearly $4000 \mathrm{H}$ or $8100 \mathrm{~Hz}$. 


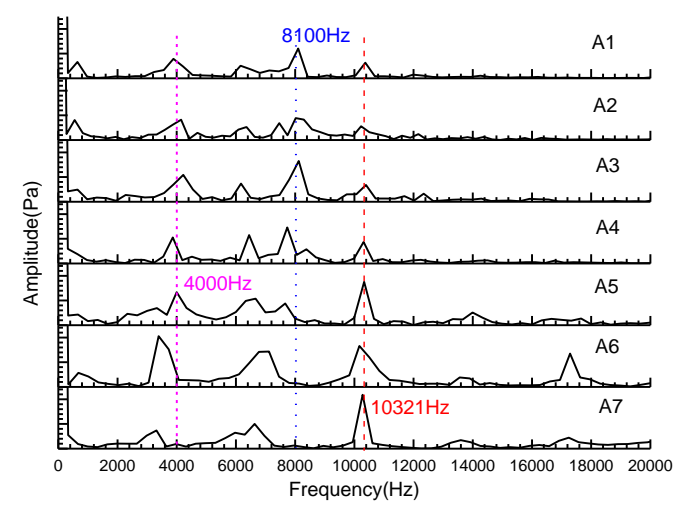

Figure 5 Frequency spectrum results of probe supports wake

\section{Unsteady Results from Mid-span Surface}

The compressor working point A1, A5 and A7 is selected for unsteady flow structure analysis. The static entropy contours of 50\% span surface are presented at a series of three time instants at fifty time step intervals in Fig.6. It is shown that the vortexes shedding from inlet probe support play an important role in changing flow structure of compressor. The vortexes are forced to shift in the direction of rotor rotation as they travel through the downstream blade row. In the following analysis, the vortex shedding from the right side of support (View from inlet of calculation domain) is marked as "Vortex 1" and the vortex shedding from the left side of the support labelled as "Vortex 2". The stator passages in calculation domain are marked as "PS1, PS2, PS3" from the bottom to the top in Fig.6

In the process of shedding vortex developing to the downstream, affected by flow diffusion and forced by the rotor blade, vortex moves along the circumferential direction. As shown in Fig. 6, when the compressor working at the choke point A1, the shedding vortex of the probe support mainly affects the flow structure of the stator passage PS2. But when the compressor working at the near-stall points A5 and A7, the flow fields in stator passages PS2 and PS3 are affected by the shedding vortex of probe support. First, analyse the near-stall points A5 and A7, where the shedding vortex frequency of the probe support is the same as the rotor passing frequency. Nolan et.al (2011) found that in rotor passage, the upstream wake vortices at the same frequency as the rotor passing would migrate circumferentially at the same circumferential speed as the rotor. Since the passing period of rotor blade and stator blade is 4:3, the Vortex 2 diffuses in the direction of rotor rotation before enter the rotor blade passage and finally develops about two stator pitches along the circumferential direction before enter in stator passage PS3. Similarly, the Vortex 1 diffuses in the opposite direction of rotor rotation and develops about one stator pitch along the circumferential direction and enters the stator passage PS2. Then to analyse the choke point A1, the wake vortex size of the probe support is larger than the working condition A5, and the corresponding tangential force of the rotor makes the vortex migration speed in the tangential direction smaller. The Vortex 1 and 2 only shifts about one stator passage and enters into PS2.
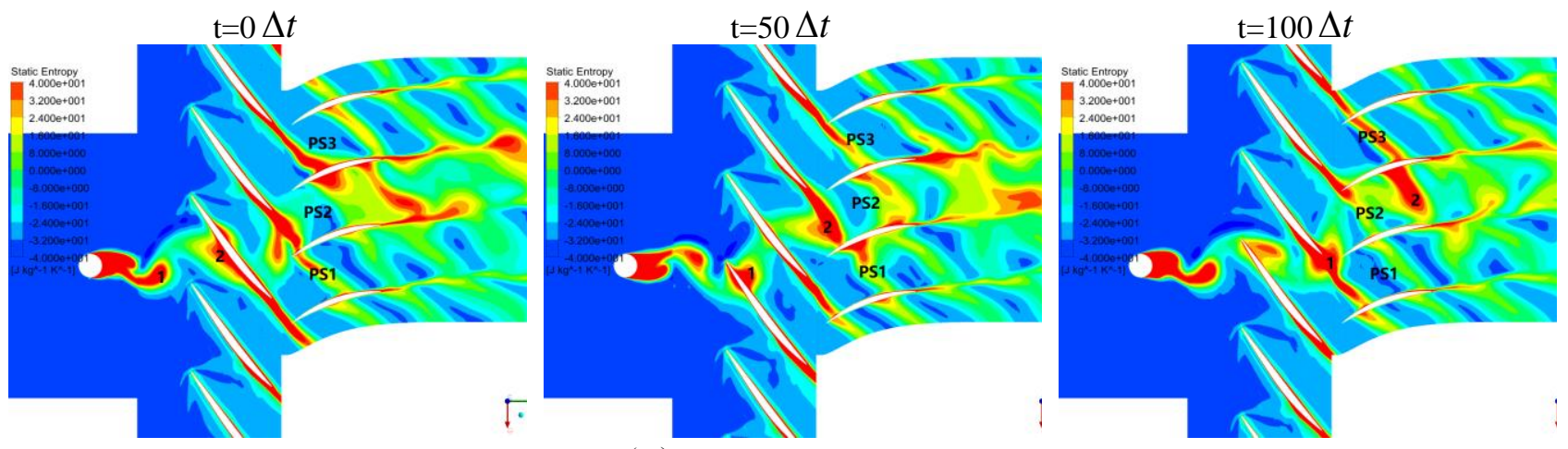

(a) Operation point A1 


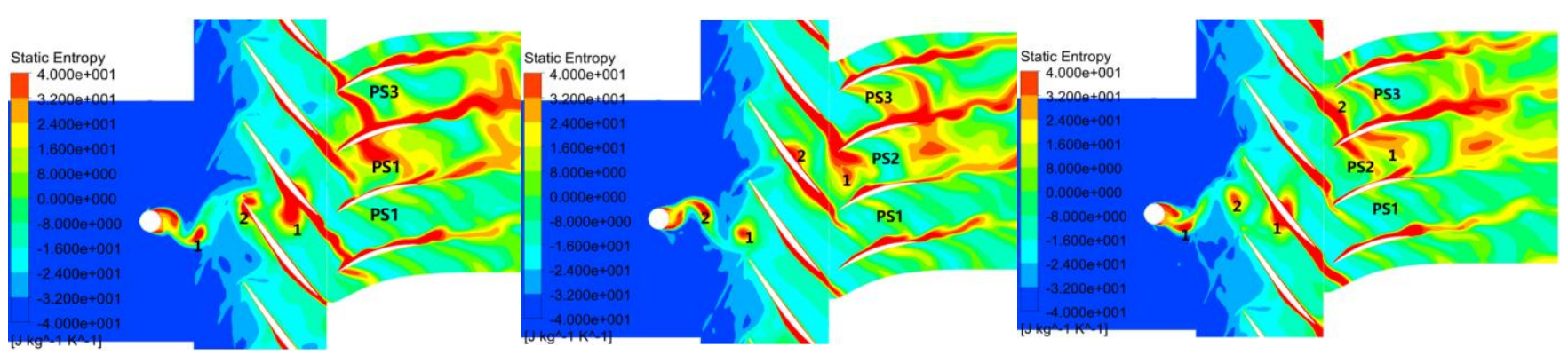

(b) Operation point A5
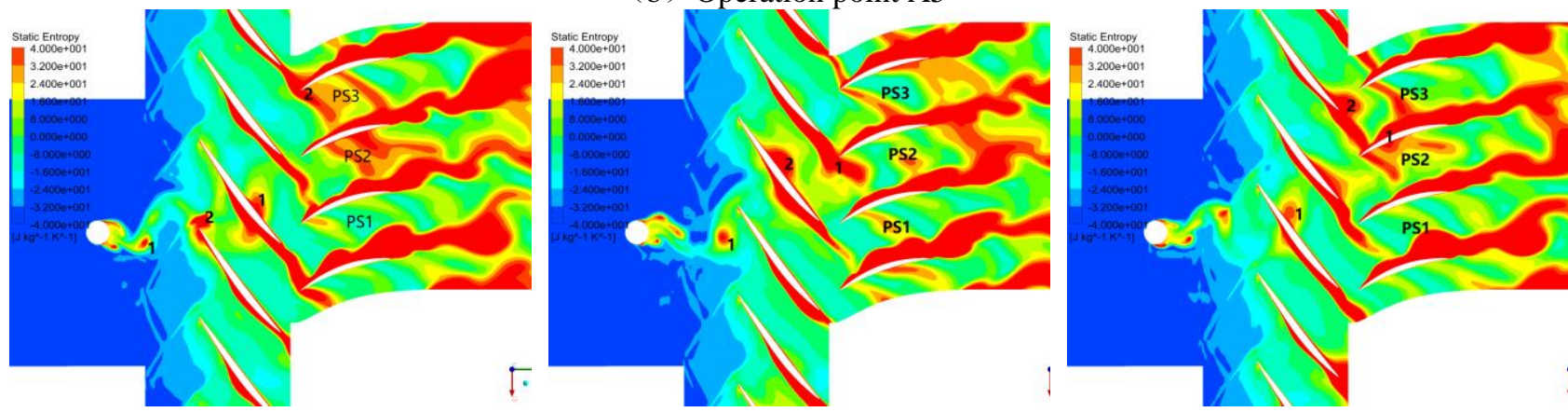

(c) Operation point A7

Figure 6 Static entropy contours of $50 \%$ span surface at three time instants

Unsteady Results from Different Span Surface

The instant static entropy for compressor working point A5 is shown on a $20 \%, 50 \%$ and $80 \%$ span surface respectively in Fig 7. The difference in phase of straightened supports and the twisted blades makes the support produce shedding vortexes in different phases. With the effect of shedding vortexes, the flow field of PS2 alters at blade tip and root, but the flow field of both PS2 and PS3 is changed notably at blade mid span. The support produces the weakest shedding vortexes at blade root (20\% span), which leads to the changes in the flow structure of the stator PS2 slightly. And the flow varies significantly in stator passages at the mid and tip (50\% and $80 \%$ span) of blades due to the enforced strengthen of shedding vortexes. At the blades tip, Vortex 1 and Vortex 2, the largest in size and strengthen, coexist in one stator passage and cause great change in the flow structure of the stator, especially for PS2.

Because the rotor blade is twisted and sweptback, the distance between support trailing edge and rotor leading edge increases from blade root to tip. The longer distance the support and rotor have, the more time the shedding vortexes need to covert across the gap, affecting the relative location of shedding vortexes and blade leading edge at rotor inlet plane. At $20 \%$ and $80 \%$ span-wise section, the vortexes shedding from support get into the rotor passage from mid-pitch of the rotor. At mid-span section, however, the vortexes enter the rotor passage from the blade leading edge. Therefore, the tangential velocity of Vortex 2 reaches the maximum when moving along with rotor suction surface, but achieves a minimum when passing through the mid pitch of downstream rotor. The Vortex 2 goes into the PS3 at 50\% span surface, and it enter PS2 at $20 \%$ and $80 \%$ span surface.

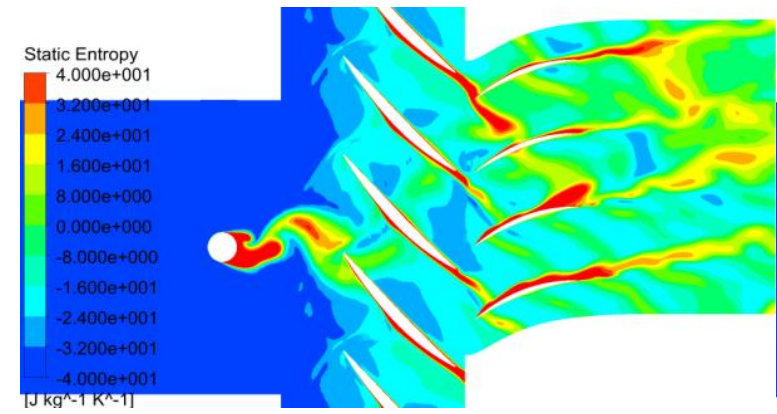

(a) $20 \%$ span

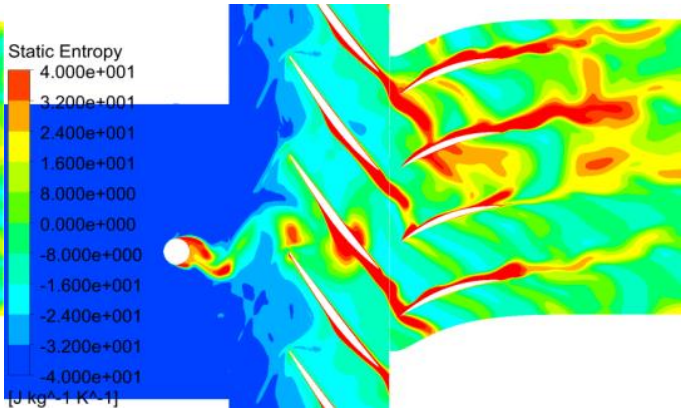

(b) $50 \%$ span 


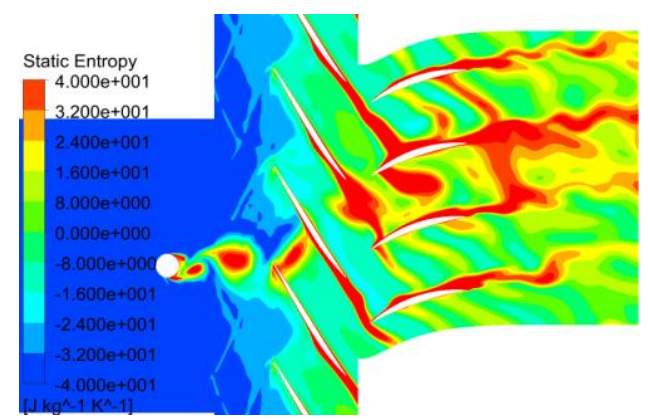

(c) $80 \%$ span

Figure 7 Instantaneous static entropy contours of $20 \%, 50 \%, 80 \%$ span surface at operation point A5

\section{CONCLUSIONS}

In this paper, a transonic compressor NASA Stage35 without IGVs but with inlet probe supports is studied using the unsteady numerical method. Conclusions are drawn as follows:

(1) Inlet probe supports has a negative impact on the overall performance of the compressors.

(2) The shedding vortex frequency of probe support is related to the working condition of the compressor. When the compressor is operating at the near-stall point, the strong interaction between probe support and rotor caused by the detachment leading edge shock, result in the shedding vortex frequency locked to the rotor blade passing frequency. Otherwise, the shedding vortex frequency is determined by Strouhal's number.

(3) The shedding vortex of the probe support affects the specific number of the downstream stator passages, which depends on the vortex transport speed along tangential direction in the rotor. The more circumferential transport speed of vortex is, the more stator passages are affected. When the shedding vortex frequency of probe support and the rotor passing frequency are locked, the vortex transport speed in the tangential direction is equal to the rotor rotation speed, otherwise it is less than the rotation speed of the rotor.

(4) From blades root to tip the scales and strengthen of shedding vortexes from the probe supports increase under the influence of rotors. Meanwhile, the relative position of shedding vortexes and rotor blade leading edge at rotor inlet plane vary along the span-wise direction because of different spacing between the probe supports and rotor blade.

\section{REFERENCES}

Zhang Baocheng (2005), Aero engine test and test technology. Beijing University of Aeronautics and Astronautics Press. J. S. Wyler (1975), Probe Blockage Effects in Free Jets and Closed Tunnels, Journal of Engineering for Gas Turbines \& Power, 97, pp.509-515.

Jan Lepicovsky (2008), Effects of a Rotating Aerodynamic Probe on the Flow Field of a Compressor Rotor, Technical Report, NASA/CR-2008-215215

Ma H, Li S, Wei W (2014), Effects of probe support on the flow field of a low-speed axial compressor, Journal of Thermal Science, 23, pp.120-126.

LJ Goldman, RG Seasholtz, and KL Mclallin (1976), Velocity surveys in a turbine stator annular-cascade facility using laser Doppler techniques, Technical Report, NASA TN

Coldrick S, Ivey P, Wells R (2002), Considerations for using 3D probes in high speed axial compressors, ASME Turbo Expo 2002: Power for Land, Sea, and Air., pp227-234.

Yang Rongfei, Zhao Jiantong, et al. (2017), Effect of inlet probe support on aerodynamic performance of 1.5-stage compressor, Journal of Propulsion Technology, 38, pp.1038-1046.

Reid L., Moore R. D. (1978), Performance of Single-stage Axialflow Transonic Compressor with Rotor and Stator Aspect Ratios of 1.19 and 1.26, Respectively, and with Design Pressure Ratio of 1.82, Technical Report, NASA-TP-1338.

Xiang, H. , Ge, N. , Hou, M. , \& Gao, J. . (2017). An experimental investigation of the effects of upstream probe disturbance on compressor cascade performance. Proceedings of the Institution of Mechanical Engineers Part G Journal of Aerospace Engineering, 231(G7), 1248-1257.

Morini, M., Pinelli, M., Spina, P. R, Venturini, M (2010). Computational Fluid Dynamics Simulation of Fouling on Axial Compressor Stages, Journal of Engineering for Gas Turbines and Power, 132, pp 072401-1-072401-10.

Dale Van Zante, Jenping Chen, Michael Hathaway, et al.(2008), The influence of compressor blade row interaction modeling on performance estimates from time-accurate, multistage navier-stokes simulations. Journal of Turbomachinery, 130, pp.011009-1 011009-10

S. P. R. Nolan, B. B. Botros, C. S. Tan (2011), Effects of Upstream Wake Phasing on Transonic Axial Compressor Performance, J. Turbomach, 133(2): 021010. 